\title{
Comparative Analysis of Packet Delay based on Density of Network
}

\author{
Harshita Tuli ${ }^{\# 1}$, Sanjay Kumar ${ }^{* 2}$ \\ ${ }^{\text {\# }}$ Research Scholar \\ Computer and System Sciences, Jaipur National University \\ Email ID: Harshitatuli@gmail.com \\ *Associate Professor \\ Computer and System Sciences, Jaipur National University \\ Email ID: Sanjaysatyam786@gmail.com
}

\begin{abstract}
MANETS are composed of sensing nodes which organize themselves into temporary topologies and forward data to each other. Delay in packet delivery depends on interference from various nodes in the network, length of path from source to destination, hop count, mobility of nodes, interference from other neighbours, bandwidth etc. To make data delivery more reliable, accurate prediction of delay is necessary task. The goal of this paper is to predict the source to destination delay in MANETs in presence of all the factors affecting the delivery of packet and to compare the prediction results in case of different protocols when the size of network also varies from sparse to dense.
\end{abstract}

Keywords- MANET, NS3, Artificial Neural Network model; ANN, Time series prediction model, AODV protocol, delay prediction, Quality of Service; QoS, FlowMonitor, Network Monitoring.

\section{INTRODUCTION}

The sensor nodes moving in sensor field and forming a special network known as Mobile Adhoc network (MANETs). All nodes can voluntarily and dynamically self-organize into arbitrary and temporary "Ad Hoc" network topologies. [1] Since the nodes are mobile and changing locations rapidly, they will be entering or leaving the sensor area very fast. Also, if two nodes within their transmission range will have a direct path between them they can communicate directly but they must communicate with each other using a number of links through different intermediate hops if they are not within each other's transmission range. The network is ad-hoc because selection of node that forwards data will be chosen dynamically based on network connectivity. This implies that every node performs as a host as well as a router since it oversees routing information between its neighbours. For such an erratic network, routing is much more complex than in traditional wireless systems, due to the lack of integrated control and information about prearranged topology. The lack of infrastructure makes it difficult to ensure the reliability of packet delivery over multi-hop routes. Because of challenges associated with routing of packets in the past they have always been the area of research for the scholars. Many research activities have been conducted to enhance the efficiency of protocols of different classes. Because of the dynamic nature of MANETs end to end stable communication and assurance of packet delivery has always been at threat. [2] Thus, in a MANET, quality of service is of prime concern. Quality of Service (QoS) refers to the capability of a network to provide better service to selected network traffic over various technologies. To provide quality delivery to real time applications, it is imperative that ad-hoc networks provide quality of service (QoS) support in terms of throughput, delay, jitter, reliability etc. There are many researchers who have tried to address the issue of QoS many times through many ways. In this paper, we have tried to focus on delay which is an important QoS metric in adhoc networks routing. A component of the delay which does not vary from packet to packet can be ignored, hence if the packet sizes are the same and packets always take the same time to be processed at the destination then the packet arrival time at the destination could be used to determine the delay component. Delay is summation of transmission, propagation and processing delay. Transmission of delay depends on interference because of many nodes in the network. Estimation of end to end packet delay in MANET is complex because transmission, queuing and processing delays have to be considered, which in turn depends on many factors such as path length from source to sink, intermediate hops, link expiration time, mobility and interference etc. In this paper, we have tried to determine the total delay sum of packets from source to destination in presence of all the factors affecting delay. To check the accuracy of prediction model we have made the network dense in a scenario and sparse in the other one. We have used ANN time series prediction model to predict the delay. The paper is categorized into different sections like background work, data collection, ANN model, conclusion \& future work and reference section. The conclusions of the comparisons are mentioned in the later sections. 


\section{BACKGROUND}

Delay is an important metric of Quality of service which is not independent. It depends on many parameters of the network for its determination. The basic reason of delay in packet delivery is nature of MANETs. So MANETs are the victim and also culprit for untimely delivery of data packets or loss of data packets. Also, Delay is summation of transmission, propagation and processing delay. The processing delay is the delay between times the packet is correctly received at the head node of the link and the time the packet is dedicated to an outgoing link queue for transmission. Processing delay component is studied in [3]. Queuing delay is a direct sign of congestion level of the network. They have suggested that as number of packets to be transmitted at each node is different, therefore the average number of packets in the queue and the associated queuing delay at each node is different. Also from this paper it has been observed that queuing delay is directly proportional to traffic which in turn directly depends on Collison of packets. The transmission delay between the times that the first and last bits of the packet are transmitted. In other words, Transmission delay can be termed as summation of Queuing delay and propagation delay depends on mobility of nodes in a network. Transmission delay can also be defined as function of the link capacities along the path, as well as the packet size. Transmission delay is quantified by transport capacity which is determined by multiplying bits and the distance per second and throughput capacity which is expressed simply by bits per second. [4] Medium access delay comprises of the latencies for data transmissions and retransmissions. This type of delay occurs if the packet is lost or sending is not completed because of different problems over the network. The data packet is sent and resent again and again if source doesn't receive the acknowledgement of the sent packet. And as MANETs are highly dynamic networks, packets can be lost due to many reasons like dynamic topology, bandwidth issues etc. The end to end packet delay in mobile ad-hoc network is complex because it depends on many influential factors as path length from source to destination, average neighbours of intermediate hops and interference. [5][6]

On the basis of available papers, it has been review in [7] that delay is a dynamic metric which is built upon the following factors multi-hop nature of network, Mobility, path length, Dynamic topology, Link-Breakage, limited battery life, bandwidth issues and Selfish behaviour of nodes. And in a dense network these factors show their affect even more than in a sparce network. A path is the systematic list of links between a source and destination. It has an account of all the hops on the route. The total number of links between a source-destination pair defines the path length between that pair. A paper written by JP singh et.al have tried to build an analytical relationship between end-to-end delay, path length and previous delay. Path length and end to end delay has been established by regression analysis. The end-to-end delay is also represented as a fuzzy time series. [8] The current end-to-end delay is then obtained by collecting regression equation of predicted delay and fuzzy time series. Mansour Sheikhan and Ehsan Hemmati,in 2011 has done a study on a transient chaotic neural network (TCNN) and proposed a multipath routing algorithm in MANETs in which it has been nominated that each node in the network should be equipped with a neural network, and all the network nodes should be trained to obtain optimal disjoint paths of great reliability. In this paper, the performance of the proposed algorithm is correlated with shortest path algorithm; disjoint path set selection protocol algorithm, and Hopfield neural network (HNN)based model. A highly dynamic topology is a distinguished feature of MANETs which poses a great challenge before them. Links between nodes are vigorous and are created and broken, as the nodes move within the network. Node mobility affects not only the transmission from source to destination, as in a conventional wireless network, but also intermediate nodes, due to the multihop nature of networks. The resulting routes can be extremely volatile, making successful ad hoc routing dependent on topological changes. In order to better understand this environment, a number of characteristics concerning the links and routes that make up an ad hoc network are studied. [9] Several other network parameters are examined in 2005 by Bren Ishibashi et.al; including hops count, network range, and radio transmission range, as well as mobility parameters for maximum speed and wait times. Also, it is assumed in this paper if topology is controlled transmission power can be adjusted to minimize the interference among the nodes. [10] In 2015 Zhang et.al; proposed an interferencebased topology control algorithm so that the network can be constrained in terms of delay. The objective of the algorithm is to adjust the transmission power to minimize interference, which proves to be a contradiction to the requirement of delay constraint adhoc networks. As When transmission power of nodes is increased to reduce the delay, interference from other active nodes in the transmission range in the network also increases. They have proposed an algorithm to establish a relation between reducing delay and minimizing interference. The proposed algorithm controls the topology to satisfy the interference constraint, and increases the transmit range to meet the delay requirement. There are many challenges associated with MANET but most importantly mobility of nodes is the biggest reason for delay in transmission. Because of nodes mobility, some of links may fail as soon as the path is established. And greater the number of nodes in the network higher is the mobility of nodes which in turn increases the dynamic nature of network. Links between nodes are vigorous and are created and broken, as the nodes move within the network. Because of nodes mobility, some of links on the shortest path may fail as soon as the path is established. [12] (Dervis Karaboga et al., 2012) This failure causes connection interruption and data loss, if the routes phase is not rediscovered rapidly. Link breakage is the basic consequence of mobility of nodes. Nodes on the path move randomly to different positions making the links 
vulnerable and dynamically changing. Link availability is used to measure probability or degree that a link is available. There are many researchers who have worked on the link quality prediction so that while routing path should be chosen depending upon the lifetime of link between hops. Palma et al., 2012[13] and chintawar et al., 2011[14] have focused on link quality prediction and estimation. From above literature synthesis, it has been observed that none of the factors of delay can stand alone. Although they are interdependent, but mobility of nodes is the basic and foremost reason for other factors to occur. Due to node mobility, the topology of the network changes as fast as the nodes move in and out of the network area. Because of dynamic topology path length vary, every instant as the nodes move. Which is in turn become the basic reason for link breakage. As, the nodes are moving from one network to another bandwidth issue that is available to nodes can be deduced as the reason for selfish behaviour of nodes. Also, MANETs are multi-hop in nature which implies that packet that starts from source node will have to meet each hop on the path that leads to destination. Nodes misbehave, they accept the packet but refuse to forward the packets to save their limited battery, which results in packet dropping problem. Also, they have limited battery, which also increases their selfishness to forward the packet. Because of all these reasons packets may never reach the destination or are delayed. After reviewing the literature, it has been observed that mobility is the most crucial factor affecting delay in packet delivery. And greater the number of nodes in the network higher the chances of their movement which in turn makes the network highly dynamic. So, the focus of this paper is on the affect that density of a network will pose on delay in packet delivery.

\section{III.SETTING UP A NETWORK}

A set of experiments were performed to collect the data set. For the collection of data set the network of mutable nodes were deployed and the transmission and reception of packets was simulated. This simulation is done on NS3. Nodes are deployed in an area of 300x1500sqm. Nodes of the network are moving in the simulation area with RandomWaypoint mobility model with a speed of $20 \mathrm{~m} / \mathrm{s}$. Routing protocol used is AODV protocol. For comparison purposes the set of flows are taken to be variable. We have considered 100 flows in first scenario and 10 flows in the other one. We have done the variations so that the accuracy of prediction results can be checked. Also, other motive was to establish the relationship between size of network and prediction accuracy. Simulation results obtained are very important which are thus used as inputs to Neural network. Data is collected after simulation which is further analysed using time series prediction model of ANN. Collection of data is done through network monitoring which is the prime task of flowmonitoring module of NS3. [15] (Kurkowski et al.,2015) Following attributes are recorded from networkmonitoring:

i. timeFirstTxPacket is the time when the first packet in the flow was transmitted;

ii. timeLastTxPacket is the time when the last packet in the flow was transmitted;

iii. timeFirstRxPacket is the time when the first packet in the flow was received by an end node, i.e. the time when the flow reception starts;

iv. timeLastRxPacket represents the time when flow reception ends i.e. the time when last packet in the flow was received;

v. delaySum represents the summation of all end-to-end delays for all received packets of the flow; (cavin et al., 2002.) [16]

here the predictions are made based on lastdelay parameter for analysis of delaysum in different scenarios.

\section{ARTIFICIAL NEURAL NETWORK MODEL FOR PREDICTION}

For predictions to be accurate the input variables are of supreme importance as they determine the structure of ANN. And moreover, time series prediction techniques can predict the values of future data set of the series based on past data observations. Pal et al., 2015[17] in their paper have said that the number of input variables have implications on results too. It may result in over fitting of data. For this paper, we have considered lastdelay as input variable in both the scenarios. MATLAB has been used for implementation of neural network last delay is used as input parameter for training the network. In this study, $70 \%$ of data set has been used for training $15 \%$ for validation and $15 \%$ for testing process. For Time series prediction and training of network is done using trainlm function. For the experiments performed in this work, NTSTOOL of MATLAB has been used. This tool is timeseries prediction tool of neural network model which on accepting the past values of the input variables predict the future value of the series. Ntstool command opens the neural network time series tool shown in figure 1 and solves a problem using a two-layer feed-forward network. NTSTOOL is timeseries prediction tool of neural network model has three diverse types of techniques to solve any problem. :-

1. NARX: - Non-Linear Autoregressive Model with external(exogeneous) input. This type of problem predicts a series $y(t)$ given $d$ past values of $y(t)$ and another series $x(t)$. It is a recurrent dynamic network. The nonlinear autoregressive network with exogenous inputs (NARX) and has feedback connections enclosing several layers of the network. The defining equation for the NARX model is

$$
y(t)=f(x(t-1), x(t-2), \ldots, x(t-d) \ldots
$$




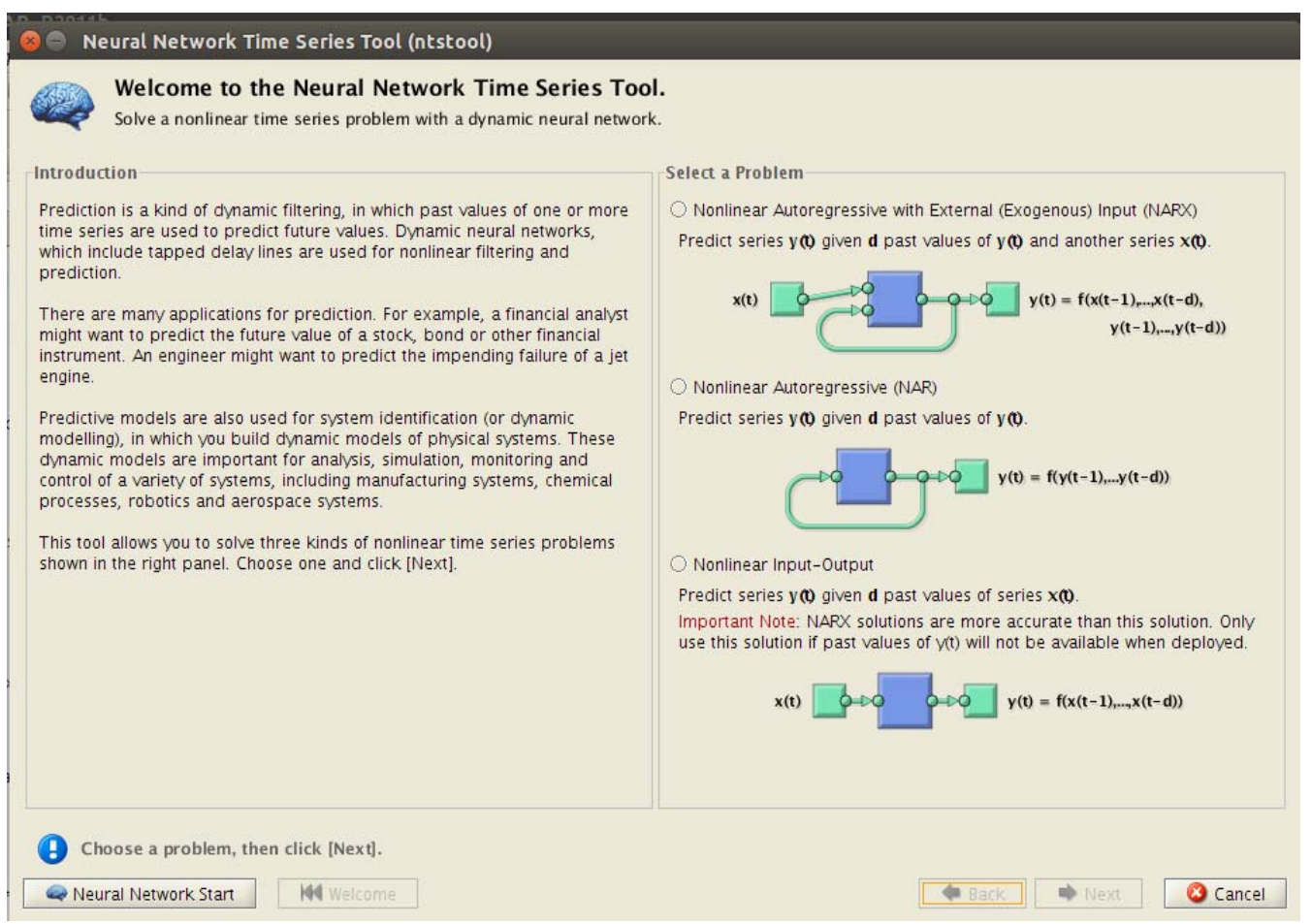

Fig 1: NARX model of time series prediction tool

where,

$\mathrm{Y}(\mathrm{t})$ is the dependent variable and its output is regressed on previous values of the output signal and previous values of an independent $\mathrm{x}$ - (exogenous) input signal.

It can also be said that $\mathrm{x}(\mathrm{t})$ is the input series on which target series i.e; $\mathrm{y}(\mathrm{t})$ series is dependents.

$\mathrm{D}$ is the delay interval of time $\mathrm{i}$ :e; time interval between two instances of input data which is recorded

\section{RESULT DISCUSSION}

Fig 2 shows the regression plot for the last delay variable as input parameter in dense network of 100 flows and Fig 3 shows the regression plot for the last delay variable as input parameter in sparse network of 10 flows. Regression values close to 1 shows clear relationship of success of training and close 0 shows that there is no relationship between predicted and actual values of parameter. In plot shown in Fig 2, the regression values for training set majorly fall on the regression line which implies that the data that has been given to the network model does not overfit. Although for validation and test data sets the scenario is not the same but if the summarisation of the plot in fourth section is to be believed then it gives the regression value to be 0.95471 which shows a close relationship between the data presented and target given.

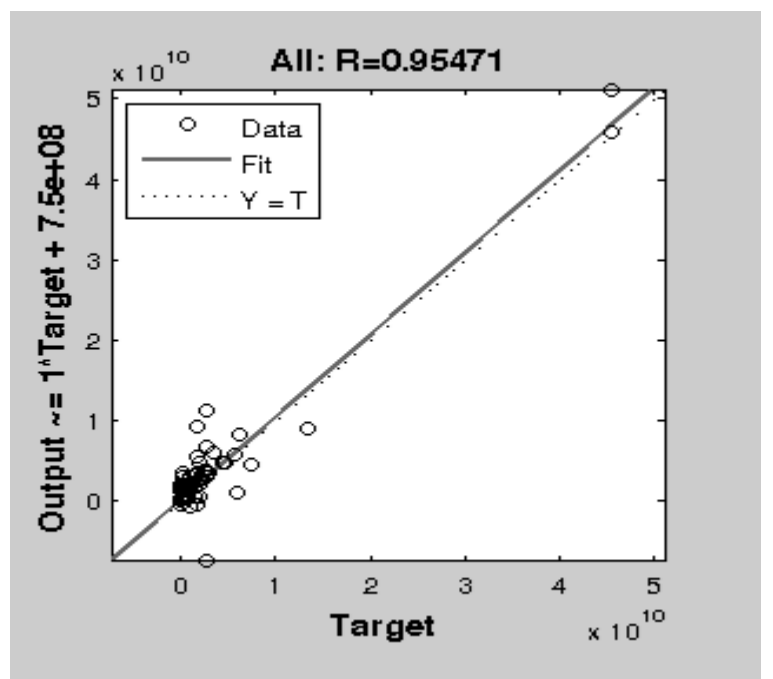

Fig 2: AODV in dense Network

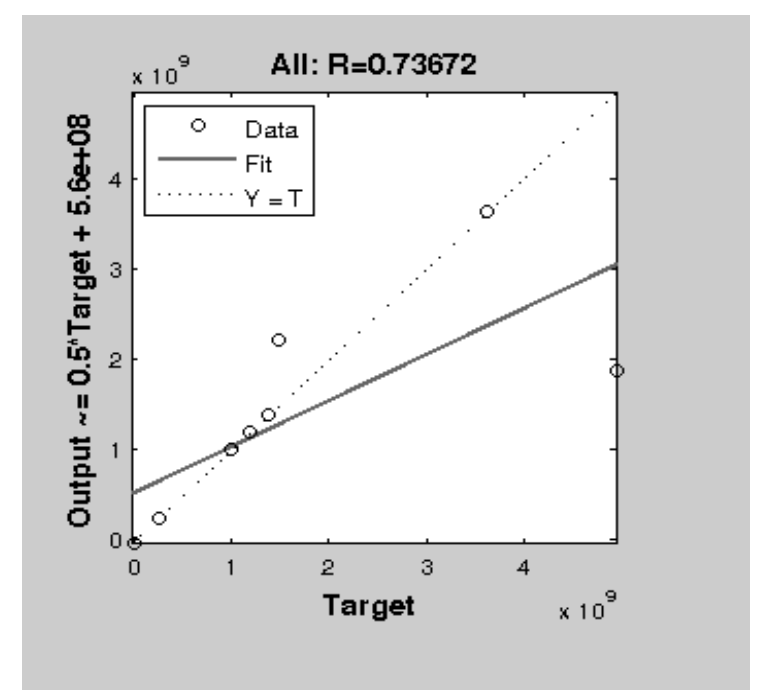

Fig 3: AODV in sparse Network 
Which implies predicted values are close to actual values of delay. Also in Fig 3 when the data is transferred using AODV protocol in a sparse network and the data is collected for training purposes and lastdelay variable from the same data is extracted and given as input to the predictor then regression values come out to be 0.73672. This shows when the network is dense the results are more accurate than in case of sparse network. Basic reason for this is lack of presented examples. When the network is sparse interactions among the nodes was less and thus presented examples to the neural network were less. Thus, the regression results are not accurate. Harshita and Sanjay in 2016 have studied a sparse network for DSDV and OLSR protocol as well and the results are stated in the table I.

TABLE I. Goodness Measure of Different Data Sets in sparse network [18] (Harshita and Sanjay, 2016)

\begin{tabular}{|c|c|c|}
\hline Sno & Protocol used for network & Regression values \\
\hline 1 & AODV & 0.73672 \\
\hline 2 & DSDV & 0.90597 \\
\hline 3 & OLSR & 0.87852 \\
\hline
\end{tabular}

\section{CONCLUSION}

In above study, we have applied autoregressive time series prediction tool of neural networks to model end to end delay of data packets in MANET. For modelling delay, we have used levenberg marquardt back propagation algorithm for time series prediction. We have considered regression plot to show the correctness of predictions, which shows that predictions done using above model in case of dense network is much closer than in sparse network. Basic reason for this is lack of presented examples to the network for learning. Future directions for the current work can be introducing the parameters to reduce the over fitting of data. Introducing other mobility models to check the patterns is another direction. Other neural network models and other soft computing techniques can be employed for better predictions.

\section{REFERENCES}

[1] Heni Kaaniche and Farouk Kamoun "Mobility Prediction in Wireless Ad Hoc Networks using Neural Networks" pub in Journal of Telecommunications, Vol 2, Issue 1, pg. 95-101 2010

[2] M. Nayak, N. Agrawal "Security in Body Sensor Networks for Healthcare applications" IOSR Journal of Computer Engineering, pg 41-46, 2013

[3] Rakesh Kumar, Manoj Misra and Anil K. Sarje "A Simplified Analytical Model for End-To-End Delay Analysis in MANET", IJCA Special Issue on Mobile Ad-hoc Networks, IJCA, pg. 195-199, 2011.

[4] Ramaswamy, Ramaswamy, Ning Weng, and Tilman Wolf "Characterizing network processing delay" Global Telecommunication Conference, GLOBECOM'04, IEEE, 2004.

[5] Syed S. Rizvi, Aasia Riasat, Khaled M. Elleithy "Quantification of Capacity and Transmission Delay for Mobile Ad Hoc Networks (MANET)", International Journal of Communications, Network and System Sciences, Research India Publications, 2010.

[6] Jyoti Prakash Singh, Prabhat Kumar, Sunil Kumar Singh "Delay Prediction in Mobile Ad Hoc Network using Trapezoidal Fuzzy numbers", Proceedings of 9th Int conf. on computer science and software engineering, IEEE, pg. 60-64,2012.

[7] Harshita Tuli and Sanjay Kumar "Review on delay prediction techniques in MANET" International journal of computer applications, vol 108, issue 14,pg. 12-14, 2014.

[8] Jyoti Prakash Singh, Paramartha Dutta, Amlan Chakrabarti "Weighted delay prediction in mobile ad hoc network using fuzzy time series”, Egyptian Informatics Journal, Elsevier, Vol 15, Issue 2, pg. 105-114, 2014.

[9] Mansour Sheikhan, Ehsan Hemmati "Transient chaotic neural network-based disjoint multipath routing for mobile ad-hoc networks" Neural Computing and Applications, Springer, Vol 21, issue 6, pg. 1403-1412, 2011.

[10] Brent Ishibashi, Raouf Boutaba "Topology and mobility considerations in mobile ad hoc networks", Adhoc Networks, Elsevier, Vol 3 , Issue 6, pg. 762-776, 2005.

[11] Xin Ming Zhang, Yue Zhang, Fan Yan, Athanasios V. Vasilakos, "Interference-based topology control algorithm for delay-constrained mobile ad hoc networks", IEEE Transactions on mobile computing, Vol 14, Issue 4, pg. 742-754, 2015.

[12] Dervis Karaboga, Selcuk Okdem, Celal Ozturk "Cluster based wireless sensor network routing using artificial bee colony algorithm" Science+Business Media, Journal of Wireless Network, Springer, Vol 18, Issue 7, pg. 847-860, 2012.

[13] David Palma, Helder Araujo, Marilia Curado "Link quality estimation in wireless multi-hop networks using Kernel based methods" Journal of computer networks, Elsevier, Vol 56, Issue 16, pg.3629-3638, 2012.

[14] A Chintawar, M Chatterjee, A Vidhate "AOMDV-APLP: An Enhanced Algorithm with Accessibility and Link Breakage Prediction", Proceedings of International Conference \& Workshop on Emerging Trends in Technology, ACM, pg. 841-845 2011.

[15] Stuart Kurkowski, Tracy Camp, Michael Colagrosso "MANET Simulation Studies: The Incredibles Mobile Computing and Communications Review", survey work supported in part by NSF Grants ANI-0208352 and ANI-0240558, Volume 1, Number 2, 2015.

[16] David Cavin, Yoav Sasson, Andre Schipre "On Accuracy of MANET simulators" Distributed System Laboratory, ACM, 2002.

[17] Arindrajit Pal, Jyoti Prakash Singh, Paramartha Dutta (2015) "Path length prediction in MANET under AODV routing: Comparative analysis of ARIMA and MLP model" Egyptian Informatics Journal, Elsevier, Vol 16, issue 1, 2015.

[18] Harshita Tuli and Sanjay Kumar "Prediction analysis of delay in transferring the packets in ADHOC networks", proceedings of IndiaCom computing for sustainable global Development, IEEE, pg. 660-662, 2016. 


\section{AUTHOR PROFILE}

Harshita Tuli received her master's degree in computer application from GGSIPU, Delhi, India in 2010. She is currently pursuing her Ph.D. degree from school of computers and system sciences, Jaipur National University, Jaipur, Rajasthan. Her research area is wireless Networking, Adhoc network and Artificial Neural Network.

Sanjay Kumar is currently working as an Associate Professor school of computers and system sciences, Jaipur National University, Jaipur, Rajasthan and has many papers to his credit. His areas of research are Artificial Neural Network, Artificial Intelligence, Machine Learning 